\title{
Separately contacted edge states: A spectroscopic tool for the investigation of the quantum Hall effect
}

\author{
A. Würtz ${ }^{a, b}$, R. Wildfeuer ${ }^{b}$, A. Lorke ${ }^{a, b}$ \\ ${ }^{a}$ Laboratorium für Festkörperphysik, Gerhard-Mercator-Universität, \\ Lotharstr. 17, D-47048 Duisburg, Germany \\ ${ }^{b}$ Sektion Physik and Center for NanoScience, Ludwig-Maximilians-Universität München, Geschwister-Scholl-Platz 1, D-80539 \\ München, Germany \\ E. V. Deviatov, V. T. Dolgopolov \\ Institute of Solid State Physics, Chernogolovka, Moscow District 142432, Russia
}

(February 28, 2022)

\begin{abstract}
Using an innovative combination of a quasi-Corbino sample geometry and the cross-gate technique, we have developed a method that enables us to separately contact single edge channels in the quantum Hall regime and investigate equilibration among them. Performing 4-point resistance measurements, we directly obtain information on the energetic and geometric structure of the edge region and the equilibration-length for current transport across the Landau- as well as the spin-gap. Based on an almost free choice in the number of participating edge channels and their interactionlength a systematic investigation of the parameter-space becomes possible.
\end{abstract}

\section{INTRODUCTION}

The concept of one-dimensional current-carrying edge channels in the quantum Hall regime has been the subject of detailed experimental and theoretical studies in recent years. It is now widely accepted as a basic ingredient for the understanding of the quantum Hall effect [1 7 . In the edge state picture, the resistance quantization is attributed to current transport in quasi one-dimensional edge channels along the sample boundaries, assuming negligible backscattering between opposing edges of the two-dimensional electron gas (2DEG) due to vanishing bulk conductivity [4,5]. Edge channels are formed at the intersection of Landau-levels with the Fermi-energy, where the presence of unoccupied states allows for current transport. Theoretical treatments, such as the compressible/incompressible liquid picture [6,7] have led to a better understanding of the energetic structure of the 2DEG-edge. Various efforts have been made to experimentally investigate the details of edge-reconstruction and edge channel transport by means of tunneling- [8:9] or capacitance-spectroscopy [10]. At the same time advances in far infrared- [11 13], time-resolved transport[14 and edge-magnetoplasmon-spectroscopy [15], as well as SET-measurements 16 have given further insight in the edge structure of the 2DEG. However, the experimental investigation of the potential profile at the sample boundaries remains a challenging task.

Here we report on the development and application of a new sample geometry, which renders possible a direct measurement of the charge transfer between adjacent edge channels at the sample boundaries. The combination of a quasi-Corbino 17 topology and the cross-gate technique [18,19] has a number of advantages, which make it a versatile tool for studying edge channel transport: Requiring no sophisticated sample fabrication such as high-resolution lithography or cleaved-edge overgrowth 20], it offers true 4-probe measurements in both, the linear and the non-linear transport regime. Furthermore it can "dissect" the edge channel structure, i.e. for $n$ edge channels, anyone of the $n-1$ gaps can individually be addressed. This is done by separately contacting single edge channels, selectively populating them [21,22] and bringing them into a controlled interaction. We present experimental data obtained using this geometry which allows us to determine the equilibration-length in the linear regime and get information on the energetic edge structure (i.e. the spin- and Landau-gaps) by non-linear $\mathrm{I}-\mathrm{V}$-spectroscopy.

This report is organized as follows: Section II gives a detailed description of the sample geometry, and the general concept behind the experiment. Also the application of the Landauer-Büttiker formalism [4, to the given topology is briefly outlined. Experimental results for different filling factor combinations, temperatures and interaction-lengths are presented in Section III. These will be discussed in terms of the edge-reconstruction picture and evaluated with regard to the spin- and Landaugap structure.

\section{DEVICE GEOMETRY}

\section{A. Concept and realization}

The samples were fabricated from a molecular beam epitactically-grown GaAs/AlGaAs heterostructure, consisting of a $1 \mu \mathrm{m}$ GaAs buffer layer, $20 \mathrm{~nm}$ undoped 
$\mathrm{Al}_{0.3} \mathrm{Ga}_{0.7} \mathrm{As}, 25 \mathrm{~nm}$ silicon-doped $\mathrm{Al}_{0.3} \mathrm{Ga}_{0.7}$ As covered by a $45 \mathrm{~nm}$ superlattice and cap-layer. The mobility at liquid Helium temperature was $800000 \mathrm{~cm}^{2} / \mathrm{Vs}$ and the carrier density $3.7 \cdot 10^{11} \mathrm{~cm}^{-2}$. All structures were patterned by standard photolithography. The mesa was defined by wet chemical etching. The gate-electrode consists of $5 \mathrm{~nm}$ thermally evaporated $\mathrm{NiCr}$ (1:1). Ohmic contacts with typical resistances of $200 \Omega$ at $30 \mathrm{mK}$ are provided by alloyed AuGe/Ni/AuGe (88:12) pads.

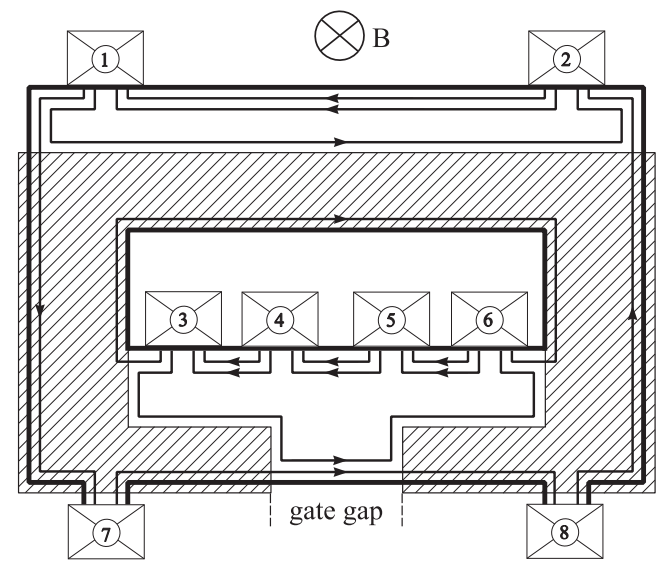

FIG. 1. Schematic diagram of the device geometry. Contacts are positioned along the inner and outer edge of the ring-shaped mesa. The shaded area represents the Schottky-gate. Arrows indicate the direction of electron drift in the edge channels for the outlined configuration: filling factor $\nu=2$ in the ungated regions and $g=1$ under the gate.

For the measurements reported here a total of 4 samples with slightly different geometries, fabricated from the same heterostructure, were investigated. The device geometry is given in Fig. 1, where the ring-shaped mesa is shown by the thick outline and the gate-electrode is indicated by the hatched area. In a quantizing magnetic field and for zero gate voltage, the present quasi-Corbino geometry has two sets of edge channels, called "inner" and "outer" edge states in the following. The shape and location of the gate is chosen such that, by application of a suitable negative bias, the inner edge channels can be redirected to run along the outer edge in the gate-gap region. This situation is shown in Fig. 1 for equilibrium conditions, i.e. without an external applied current. Here, the filling factors have been adjusted to $\nu=2$ in the ungated regions and $g=1$ under the gate. In the gate-gap there are two adjacent channels running in parallel. One of them is reflected by the gate potential, and connected only to the inner Ohmic contacts. The other, outer edge channel continues to run along the etched boundary, even under the gate-electrode, and is therefore connected only to the contacts positioned along the outer mesa edge. When the bulk region of the 2DEG under the gate is in its insulating state (integer filling factor and sufficiently low temperatures) current trans-

port between inner and outer Ohmic contacts is possible only by charge equilibration among neighboring channels in the gate-gap region. The present geometry therefore allows for a direct investigation - in 2- or 4-probe geometries - of transport across the incompressible strip that separates the compressible edge channels within the gate-gap.

$$
\nu=2 \quad \nu=3 \quad \nu=4
$$

$g=1$
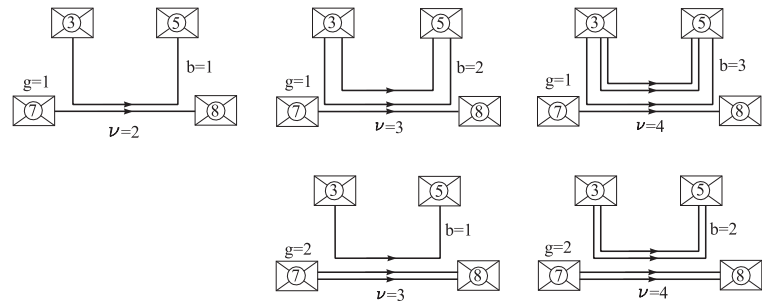

$g=3$

$\otimes$ B

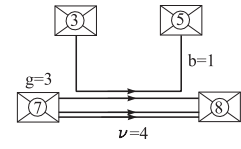

FIG. 2. Survey on the adjusted filling factor combinations in the IQHE regime. Outlined are only the traces of edge channels in the gate-gap region. Here $\nu$ indicates the number of occupied spinpolarized edge states in the ungated regions, $g$ the number of channels transmitted under the gate and $b$ the number of channels reflected by the gate potential.

Figure 2 illustrates the versatility of the present geometry. For any given integer filling factor $\nu$ all incompressible strips can be probed by adjusting the gate voltage to the appropriate integer filling factor $g$ in the gated region. When spin-splitting is resolved and the filling factors are adjusted to $\nu=4$ and $g=1$, the outermost spin-induced incompressible strip can be probed, whereas for $\nu=4$ and $g=2$ the wider, Landau-gap-induced strip between the second and third edge channel can be investigated. For clarity, in Fig. 2 only the four contacts used as current leads or voltage probes in the experiment are shown. Furthermore, only edge channels running by the interaction region (i.e. the gate gap) are drawn. In the evaluation of the experimental data it is of course necessary to take into account the complete sample geometry, as it will be discussed in the following section.

\section{B. Application of the Landauer-Büttiker formalism to the experimental setup}

Despite the apparent complexity of the device geometry, a relation between the measured resistances and equilibration based on inter-edge-channel transport (expressed in terms of an effective transmission-coefficient $T$ across the incompressible strip) can be derived in a 
straightforward manner. In the following, we will treat the case of a 4-probe measurement, restricting the discussion to the leads $3,5,7,8$. It is easy to show that the presence of the unused (floating) contacts 1, 2, 4, 6 neither changes any of the considerations below nor the obtained experimental results. Based upon the absence of backscattering across the bulk and the conservation of current, a Landauer-Büttiker-type multichannelmultiprobe formula can be derived (see Appendix A) to give the following results for the four different contact configurations for measuring the 4-probe resistance between inner and outer edge channels:

$$
\begin{aligned}
& R_{73,85}=\frac{h}{e^{2}}\left[\frac{1}{T}-\frac{\nu+g}{\nu g}\right] \\
& R_{75,83}=\frac{h}{e^{2}}\left[\frac{1}{T}-\frac{1}{g}\right] \\
& R_{85,73}=\frac{h}{e^{2}}\left[\frac{1}{T}\right] \\
& R_{83,75}=\frac{h}{e^{2}}\left[\frac{1}{T}-\frac{1}{\nu}\right]
\end{aligned}
$$

Here $R_{i j, k l}$ is the resistance measured between the current leads $i$ and $j$ and voltage probes $k$ and $l$. Note that exchanging current and voltage probes changes the value of the resistance because of the chirality of edge states $\left(R_{i j, k l}(B)=R_{k l, i j}(-B) \neq R_{k l, i j}(B)\right)$. The measurements can therefore be used to confirm the direction of electron-drift, respectively the orientation of the magnetic field in the experimental setup.

Combining equation 1 with the relation between $T$ and the equilibration-length 23] $l_{e q}$ gives for the interaction of two edge channels

$$
R_{85,73}=\frac{h}{e^{2}}\left[1+\exp \left(\frac{-2 d}{l_{e q}}\right)\right]^{-1}
$$

with $d$ being the interaction-length, i.e. the gate-gap width. This means that the present topology makes it possible to determine the equilibration-length between neighboring edge channels by a single resistance measurement.

For elevated temperatures or macroscopic interactionlengths, full equilibration is expected. Then the transmission-coefficient can be derived from a summation over all participating edge states and contacts:

$$
T=\sum_{i=1}^{g} \sum_{j=g+1}^{\nu} 1 / \nu=(\nu-g) \cdot g / \nu .
$$

In this case all the resistances $R_{i j, k l}$ are readily calculated and can be compared with the experimental values.

Finally, it should be mentioned that at no point in the derivation of equation 11 the transmission-coefficient is assumed to be independent of the electrochemical potential difference across the incompressible strip. Equilibration between edge states can therefore be studied as a function of bias. As will be shown in Section IIIB, this $\mathrm{I}-\mathrm{V}$-spectroscopy can be used not only in the linear transport regime ( $T=$ const.), but also to investigate the local energetic structure of the edge region.

\section{EXPERIMENTAL}

\section{A. Equilibration across the Landau-gap at liquid helium-temperature}

Figure 3 shows current-voltage curves obtained for a sample with $32 \mu \mathrm{m}$ gate-gap width, a filling factor combination $\nu=4, g=2$ and a temperature of $4.2 \mathrm{~K}$. The inset displays the corresponding, simplified configuration of edge channels (see also the discussion of Fig. 2). As expected from equation 1, each configuration gives a different 4-point resistance (dashed lines indicate experimental curves). For comparison, the solid lines show the resistances for complete equilibration $(T=1)$, calculated as discussed in Section II B 24].

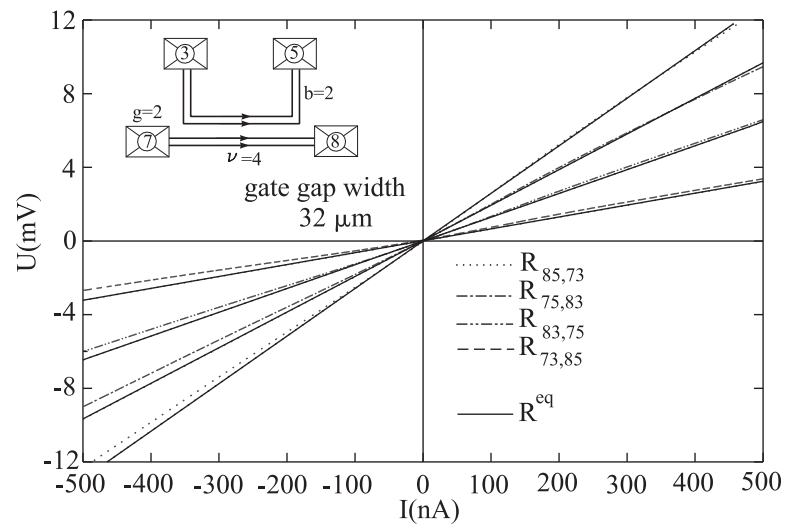

FIG. 3. Measurements at $4.2 \mathrm{~K}$ and $\mathrm{B}=3.85 \mathrm{~T}$ on a device with gate-gap width $32 \mu \mathrm{m}$ for the adjusted filling factor combination $\nu=4$ and $g=2$. The solid lines indicate the calculated resistances, according to the equations 11 and 3 for fully equilibrated transport.

The very good agreement between the calculated resistances for fully equilibrated transport and the measurements at $4.2 \mathrm{~K}$ indicates that at this temperature the equilibration-length is much shorter than the gate-gap width of $d=32 \mu \mathrm{m}$. Furthermore, the fact that equations 1 and 3 can so well account for the experimental data shows that the discussed Landauer-Büttiker picture is valid and in particular that transport through the bulk of the $2 \mathrm{DEG}$ is negligible. The absence of bulk leakage at $4.2 \mathrm{~K}$ for even filling factors is also confirmed by a direct determination of the bulk conductivity, adjusting the filling factors to $\nu=2, g=2$, and investigation of a reference-sample without a gate-gap [25]. Also the influence of non-ideal contact properties proves to be irrelevant. 


\section{B. Weak coupling among edge states at $30 \mathrm{mK}$}

When the samples are cooled down to $30 \mathrm{mK}$ in the mixing chamber of a ${ }^{3} \mathrm{He}^{4} \mathrm{He}$ dilution-refrigerator, the resistance at low bias increases dramatically. Even for an interaction-length of more than $30 \mu \mathrm{m}$, resistances of around $10 \mathrm{M} \Omega$ are observed, corresponding to macroscopic equilibration-lengths (see section III D).

Figure 1 shows I-V-traces of a sample with $5 \mu \mathrm{m}$ gategap width, obtained for the four different contact configurations and filling factors $\nu=4$ in the ungated areas and $g=2$ under the gate-electrode. As indicated in the inset, this filling factor combination corresponds to equilibration across the Landau-gap.

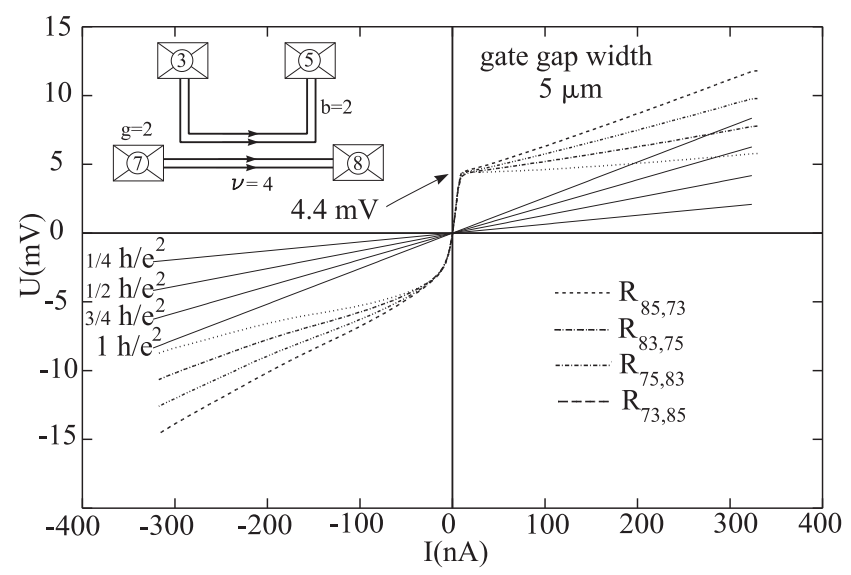

FIG. 4. Equilibration across the Landau-gap, measured at $30 \mathrm{mK}$ and $\mathrm{B}=3.89 \mathrm{~T}$. The gate-gap width of the investigated sample is $5 \mu \mathrm{m}$ and the chosen filling factors are $\nu=4$ and $g=2$. Solid lines indicate the calculated 4-point resistances for the different contact configurations according to the Landauer-Büttiker-type formulas (equations 1 and 3). The onset-voltage was determined to be $4.4 \mathrm{mV}$.

The current-voltage characteristics are strongly nonlinear and asymmetric. Regarding the low positive bias region in Fig. 1, which corresponds to a decrease of the electrochemical potential of the two outermost edge channels in the gate-gap, the occurrence of an onset-voltage is observable for all contact configurations. At biases exceeding this voltage, the experimental curves have an almost constant differential resistance. For negative applied biases, no precise identification of an onset-voltage is possible. Here, in contrast to what is observed for positive biases, the $\mathrm{I}-\mathrm{V}$-traces are not exactly reproducible from cooling to cooling and differ qualitatively from sample to sample.

At a positive current of about $10 \mathrm{nA}$, the slope of the traces shown in Fig. 4 decreases drastically and the differential resistances drop to values close to those obtained for complete equilibration. We interpret this step in the I-V-characteristic as a novel type of breakdownmechanism in the transport between adjacent edge channels, which is not related to the usual, complete breakdown of the quantum Hall effect. The latter is governed by transport through the bulk of the 2DEG, which for macroscopic samples (like the ones investigated here with lateral dimensions of a few millimeters), is only observed at much higher currents 26. In particular, no step at small voltages is found for a reference-sample with gategap widths zero, where only the breakdown through the bulk can be seen, however at much higher voltages. As discussed above, already at $4.2 \mathrm{~K}$ and even integer filling factors the $2 \mathrm{D}$ bulk is in its insulating state and no indications of breakdown are observed in the range of biases and voltages considered here (see Fig. 3). Also, bad Ohmic contacts cannot account for the step in the I-V-characteristic as the number of samples and contact combinations investigated make this explanation highly unlikely. Furthermore, in the applied 4-terminal configuration, contact resistances should not appreciably affect the results as long as the input-impedance of the experimental setup is sufficiently high.

\section{Qualitative interpretation in terms of the incompressible/compressible liquid model}

In this section we will develop a first interpretation of the experimental observations shown in Fig. \&, which is based on the breakdown across the incompressible strip [7] between adjacent, but differently biased edge channels. According to this picture it can also be understood, why the onset-voltage of about $4.4 \mathrm{mV}$, roughly corresponds to the Landau-gap $\hbar \omega_{c}$ for the given magnetic field $B=3.89$ T. Furthermore, the proposed model accounts for the asymmetry between positive and negative current directions seen e.g. in Fig. \& and tries to explain why, at sufficiently large currents the linear parts of the observed traces have almost the same slope as the fully equilibrated resistance curves (see the solid lines in Fig. 3 and the dash-dotted lines in Fig. 6, obtained from equations 1 and (2). 


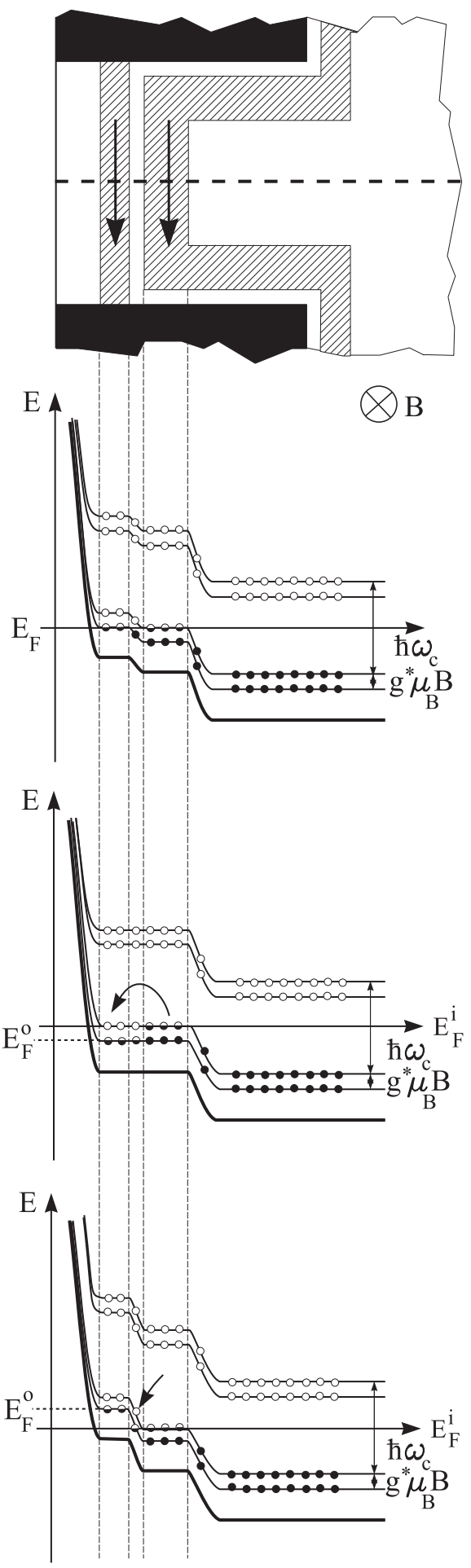

FIG. 5. Application of the edge-reconstruction model to the experimental setup. (a) Gate-gap region with compressible (shaded) and incompressible (light) liquid strips. (b) Edge potential as derived from the compressible/incompressible-liquid model in case of a constant Fermi-energy in the gate-gap region. Filled circles indicate occupied states, light ones unoccupied states. (c) Shifted potential for an applied voltage $\Delta U_{o i}=+g^{*} \mu_{B} B$ between two reservoirs at the inner and outer mesa edge. (d) Edge potential for an applied bias of $\Delta U_{o i}=-g^{*} \mu_{B} B$.
The measured I-V-spectra in Fig. 1 resemble those of a backward diode [27]. Indeed, in the picture of edgestate-reconstruction developed by Chklovskii, Shklovskii et al. [6] (taking into account screening-effects among electrons), transport across an incompressible edge-strip can be described in a manner comparable to the transport across the depletion-layer in a $p-n$ backwarddiode. This is illustrated in Fig. 5 (b)-(d), where we have sketched the edge state reconstruction for $\nu=2$, $g=1$. Also shown is the course of edge channels in the gate-gap region for this filling factor combination (Fig. 同 (a)). The mesa edge is positioned on the left hand side, shaded areas indicate compressible liquids, light regions incompressible strips and the bulk. The gate is drawn in black. Arrows indicate the direction of electron drift in the compressible liquid strips.

Figures 月 (b)-(d) show the reconstruction of the edge potential for the section indicated by the dashed line in (a). Occupied states beneath the Fermi-energy are symbolized by full circles, open circles represent unoccupied states, states at the Fermi-energy are shown as half-filled circles. In the configuration outlined in (b) no bias is applied between inner and outer contacts and no current will flow between the separately contacted edge states.

We now consider the case that one of the inner contacts is grounded and a positive voltage is applied to an outer contact. In this situation, the outer edge channel is shifted downwards in energy with respect to the inner one. For a very low bias, this aligns the occupied states in the inner channel with the energy-gap in the outer channel so that ideally no current is expected to flow. This gives reason for the appearance of the high resistance region at very small positive bias in complete analogy to the case of a backward-diode. Only when a high enough bias is applied so that the topmost occupied inner edge state becomes aligned with the first unoccupied outer edge state, transfer of electrons between the edge channels becomes energetically allowed and equilibration is readily achieved as depicted in Fig. 0 (c). This explains the sudden breakdown of the high differential resistance state when a positive voltage $\Delta U_{o i}=+E_{g} / e$ is applied between inner and outer contacts. Here, $E_{g}$ is the relevant energy-gap, which, for the case $\nu=2, g=1$ is expected to be the spin-gap $g^{*} \mu_{B} B$, with $g^{*}$ being the effective Landé-factor. 


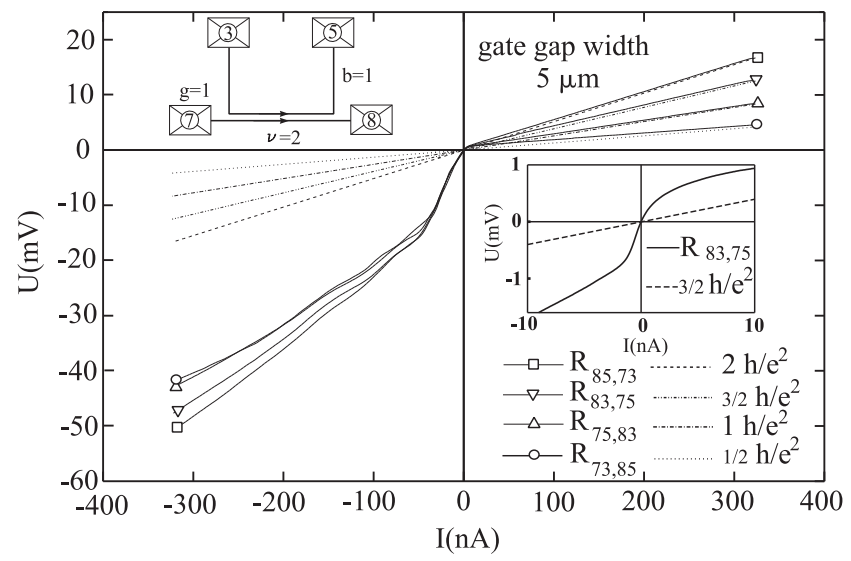

FIG. 6. Filling factor combination $\nu=2, g=1$ measured at $30 \mathrm{mK}$ and $\mathrm{B}=7.75 \mathrm{~T}$. Equilibration between inner and outer edge channels occurs across the spin-gap in the first Landau-level. Dotted lines indicate the calculated resistances for the case of full equilibration. The inset shows a magnification of the low-bias region taken for one contact combination at a low sweep rate.

For a negative bias, on the other hand, the first unoccupied inner edge strip is energetically separated from the outer occupied states by roughly the Landau-gap $\hbar \omega_{c}$ (see Fig. (d)). Therefore, a pronounced asymmetry is expected in the I-V-spectra, as it is observed experimentally for $\nu=2, g=1$ (Fig. 6).

Here, at positive voltages exceeding $1 \mathrm{mV}$, almost full equilibration is established, whereas in the negative bias regime the onset of equilibration with a higher transmission coefficient can be detected only for $|U|>10 \mathrm{mV}$. It should also be pointed out, that for a negative bias no true energy-gap opens up, since there are always unoccupied levels (arrow in Fig. 5(d)) at the quasi-Fermi-level of the outer edge channels $E_{F}^{o}$. Therefore, no clear step-like features are observed for negative biases.

Of course this model can directly be transferred to the case of equilibration across the Landau-gap $\left(E_{g}=\hbar \omega_{c}\right)$, as e.g. depicted in Fig. 1 , where the step-like features are far more pronounced and the asymmetry is still observable.

\section{Evaluation of the I-V-spectra}

The characteristic onset-voltage for equilibration across the Landau-gap is determined by linear extrapolation of the two different curve branches in the positive voltage region. For the data shown in Fig. A, we obtain $E_{g}=4.4 \mathrm{meV}$. A comparison with $\hbar \omega_{c}$ for $B=3.89 \mathrm{~T}$ and an effective electron-mass of $m^{*}=0.067 m_{e}$ shows a discrepancy of 2-3 meV. Similar deviations were observed for any of the four investigated samples and both filling factor combinations $\nu=4, g=3$ and $\nu=4, g=2$.

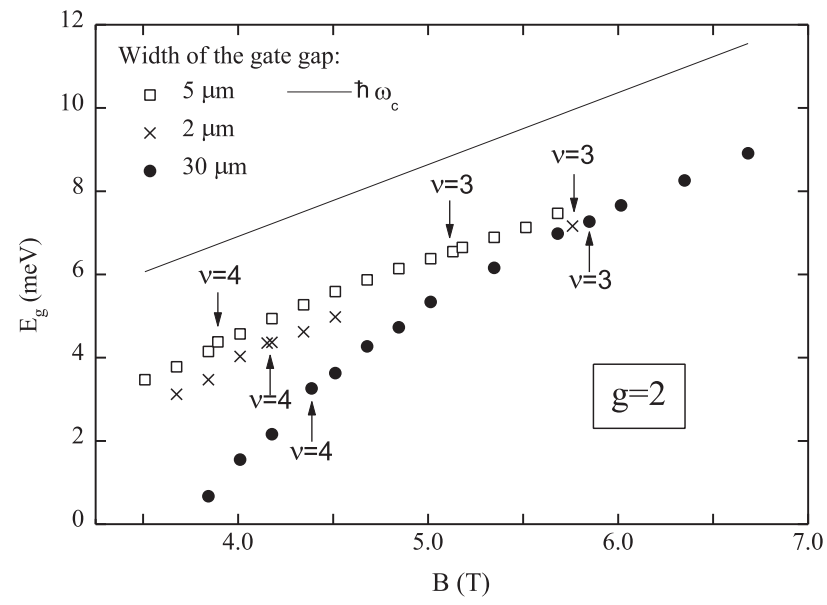

FIG. 7. Magnetic field dependence of the determined energy-gaps measured at $30 \mathrm{mK}$ for different gate-gap widths. The filling factor $g=2$ under the gate was held constant. The solid line is given by the theoretical value of the Landau-gap $\hbar \omega_{c}$.

Figure 7 shows the magnetic field dependence of the energy-gap determined for three different gate-gap widths. While the filling factor $g=2$ under the gate is held constant, the filling factor $\nu$ in the ungated areas varies with the strength of the magnetic field. Note that even though at non-integer filling factors the ungated bulk region are in a conducting state, [7] I-Vspectroscopy across the incompressible strip is still possible. The solid line indicates the calculated values of $\hbar \omega_{c}$. For $\nu \leq 4$ we obtain a linear dependence for the samples with 2 and $5 \mu \mathrm{m}$ gate-gap widths, but again the above mentioned deviation from the theoretical value of the Landau-gap is observed. A linearization of the curve slopes - where possible - allows to determine the effective mass $m^{*}$. The obtained values, as given in Table [ are in good accordance with the standard value of 0.067 $m_{e}$ for bulk GaAs.

TABLE I. Effective masses $m^{*}$ in units of the free electron mass as calculated from the slopes of the linear fits to the data shown in Fig. 月.

\begin{tabular}{lc}
\hline \hline gate-gap width & $m^{*} / m_{e}$ \\
\hline $2 \mu \mathrm{m}$ & 0.067 \\
$5 \mu \mathrm{m}$ & 0.069 \\
$30 \mu \mathrm{m}$ & 0.060 \\
\hline \hline
\end{tabular}


The deviation from $\hbar \omega_{c}$ of roughly $2 \mathrm{meV}$ can partly be understood in terms of the edge-state model discussed in section III , as the effective energy-gap width is reduced by the spin-splitting energy $g^{*} \mu_{B} B$. The Landaulevel broadening might be a further cause for the onset of equilibration at biases less than $\hbar \omega_{c} / e$. The breakdown of the adiabatic transport regime at energy values smaller but comparable to the Landau-level spacing was also reported by Komiyama et al. [28,29] for samples with a cross-gate geometry. They proposed a self-consistent reconstruction of edge states to describe the observed nonlinear behaviour, finding $\hbar \omega_{c} / 2$ to be the critical potential-difference for nonequilibrium population of edge channels.

The evaluation of the data shown in Fig. 6 gives an effective spin-gap of $0.57 \mathrm{meV}$. This corresponds to $g^{*} \approx$ 1.3 , about three times the bulk GaAs $g$-factor. Because of the smallness of the spin-gap it can only be evaluated from the present data with a large margin of error. A survey of values obtained for different gate-gap widths and filling factors (cf. Fig. 2, top row) shows a large scatter, with values from $g^{*}=0.27$ to $g^{*}=1.8$ [30]. A more thorough investigation of the parameter-space with further high-resolution measurements is therefore necessary.

Assuming a constant equililibration-rate per unitlength, the equilibration-length $l_{e q}$ can be deduced from the linear part of the I-V-curves at low positive voltages. This was done e.g. for the filling factor combination $\nu=4, g=2$. Making use of equation 1 and 2 we obtain $l_{e q}=250 \mu \mathrm{m}$ for $5 \mu \mathrm{m}$ gate-gap width and $30 \mathrm{mK}$ temperature. This corresponds to a transmission-coefficient of $\mathrm{T}=0.072$. Similar macroscopic equilibration-lengths have been reported by several authors using different approaches 23,31,28,32. Depending on the gate-gap width we found equilibration-lengths up to the order of magnitude of the sample size. Surprisingly the equilibrationlength also reveals a dependence on the choice of contacts, which is not compatible with the above discussed Landauer-Büttiker-type formulas.

\section{CONCLUSION}

In summary, we have realized a pseudo-Corbino geometry, suitable to obtain information on the energetic structure of the edge potential in the quantum Hall regime by means of simple I-V-spectroscopy. We directly observed non-linear transport across different energy-gaps and determined the transmission-coefficient for weak coupling among separately contacted edge channels. Interpreting our results in terms of the Landauer-Büttiker formalism [4,5] and the edge-reconstruction picture developed by Chklovskii et al. [6,7], we show that the described geometry enables us to shift the electrochemical potentials of single edge states with respect to each other. Transport across the incompressible liquid-strip can thereby be studied as a function of the bias applied between inner and outer contacts. Identifying the onset-voltage for inter-edge-channel current flow with the energy-gap, we found for equilibration across the Landau-gap a clear and reproducible deviation from the value of $\hbar \omega_{c}$. On the other hand, the dependence of the energy-gap on the magnetic field agrees well with the effective mass of GaAs. Furthermore, from the observed spin-gap, an effective $g$-factor can be determined, so far, however, only with a large margin of error.

As mentioned, our new spectroscopic technique has addressed a number of interesting questions regarding transport across incompressible liquid-strips and the energetic structure of the edge potential. Subject of future investigations with the proposed sample geometry will besides continuing the study of non-linear transport surely be the edge structure in the fractional quantum Hall regime as well as the influence of spin-flip processes on equilibration.

\section{ACKNOWLEDGMENTS}

We wish to thank J.P. Kotthaus for his constant support of this work and gratefully acknowledge financial support by the Deutsche Forschungsgemeinschaft, SPP "Quantum Hall Systems", under grant LO 705/1-1. We also gratefully acknowledge help during the experiments and discussions with A.A. Shashkin. The part of the work performed in Russia was supported by RFBR grant 00-02-17294, INTAS YSF002, the programs "Nanostructures" and "Statistical Physics" from the Russian Ministry of Sciences.

\section{APPENDIX A:}

Referring to the geometry shown in Fig. 11 we calculate as an example the 4 -point resistance $R_{85,73}$. Again the previously applied nomenclature is used:

$\nu$ : filling factor in the ungated regions of the sample

$g$ : filling factor in the gated regions

From the application of the Landauer-Büttikermultichannel-multiprobe formula [5] to the present geometry we derive the following "current-statistics" for the 8 shown contacts:

$$
\begin{aligned}
& I_{1}=-\frac{e^{2}}{h} \nu\left(U_{1}-U_{2}\right) \\
& I_{2}=-\frac{e^{2}}{h}\left[\nu U_{2}-(\nu-g) U_{1}-g U_{8}\right] \\
& I_{3}=-\frac{e^{2}}{h} \nu\left(U_{3}-U_{4}\right) \\
& I_{4}=-\frac{e^{2}}{h} \nu\left(U_{4}-U_{5}\right)
\end{aligned}
$$




$$
\begin{aligned}
& I_{5}=-\frac{e^{2}}{h} \nu\left(U_{5}-U_{6}\right) \\
& I_{6}=-\frac{e^{2}}{h}\left[\nu U_{6}-g U_{3}-T_{36} U_{3}-T_{76} U_{7}\right] \\
& I_{7}=-\frac{e^{2}}{h} g\left(U_{7}-U_{1}\right) \\
& I_{8}=-\frac{e^{2}}{h}\left[g U_{8}-T_{78} U_{7}-T_{38} U_{3}\right)
\end{aligned}
$$

with $T_{i j}$ being the transmission-coefficient for chargetransfer from contact $i$ to contact $j$. Taking into account charge conservation in the gate-gap region and assuming at each contact the same number of incoming and outgoing edge channels we further obtain:

$$
\begin{aligned}
T & =T_{38}=T_{76} \\
g & =T_{78}+T_{38} \\
\nu & =g+T_{36}+T_{76}
\end{aligned}
$$

Regarding now the 4 -point resistance $R_{85,73}$, i.e. current transport between the contacts 8 and 5 , with contacts 7 and 3 being used as voltage probes, we receive a surprisingly simple relation. (The currents at unused respectively floating — contacts are vanishing.)

$$
\begin{gathered}
I_{1}=0 \Rightarrow U_{1}=U_{2} \\
I_{2}=0 \Rightarrow U_{1}=U_{8} \\
I_{3}=0 \Rightarrow U_{3}=U_{4} \\
I_{4}=0 \Rightarrow U_{4}=U_{5} \\
I_{6}=0 \\
I_{7}=0 \Rightarrow U_{1}=U_{7} \\
R_{85,73} \cdot \frac{e^{2}}{h}=\frac{U_{3}-U_{7}}{I_{8}} \\
=\frac{U_{7}-U_{3}}{g U_{8}-\left(g-T_{38}\right) U_{7}-T_{38} U_{3}} \\
=\frac{U_{7}-U_{3}}{T_{38}\left(U_{7}-U_{3}\right)}=\frac{1}{T_{38}} \\
=\frac{1}{T}
\end{gathered}
$$

[1] B. I. Halperin, Phys. Rev. B 25, 2185 (1982).

[2] R. J. Haug, Semicond. Sci. Technol. 8, 131-153 (1993).

[3] A. H. MacDonald, P. Strěda, Phys. Rev. B 29, 1616 (1984).

[4] M. Büttiker, Y. Imry, R. Landauer and S. Pinhas, Phys. Rev. B 31, 6207 (1985).

[5] M. Büttiker, Phys. Rev. B 38, 9375 (1988).

[6] D. B. Chklovskii, B. I. Shklovskii and L. I. Glazman, Phys. Rev. B 46, 4026 (1992).
[7] D. B. Chklovskii, K. A. Matveev and B. I. Shklovskii, Phys. Rev. B 47, 12605 (1993).

[8] N. B. Zhitenev, R. J. Haug, K. v. Klitzing and K. Eberl, Phys. Rev. B 51, 17820 (1995).

[9] S. W. Hwang, D. C. Tsui and M. Shayegan, Phys. Rev. B 48, 8161 (1993).

[10] S. Takaoka, K. Oto, H. Kurimoto and K. Murase, K. Gamo and S. Nishi, Phys. Rev. Lett. 72, 3080 (1994).

[11] R. Merz, F. Keilmann, R. J. Haug and K. Ploog, Phys. Rev. Lett. 70, 651 (1993).

[12] A. Lorke, J. P. Kotthaus, J. H. English and A. C. Gossard, Phys. Rev. B 53, 1054 (1996).

[13] K. Hirakawa et al., The Physics of Semiconductors, M. Scheffler and R. Zimmermann, Ed. World Scientific, Singapore, 1996, p. 2543.

[14] N. B. Zhitenev, R. J. Haug, K. v. Klitzing and K. Eberl, Phys. Rev. Lett. 71, 2292 (1993).

[15] V. I. Talyanskii, A. V. Polisski, D. D. Arnone, M. Pepper, C. G. Smith, D. A. Ritchie, J. E. Frost and G. A. C. Jones , Phys. Rev. B 46, 12427 (1992).

[16] Y. Y. Wei, J. Weis, K. v. Klitzing and K. Eberl, Phys. Rev. Lett. 81, 1674 (1998).

[17] K. Oto, K. Arai, S. Uno, S. Takaoka and K. Murase, Microelectronic Engineering 47, 27 (1999).

[18] R. J. Haug, A. H. MacDonald, P. Streda and K. von Klitzing, Phys. Rev. Lett. 61, 2797 (1988).

[19] S. Washburn, A. B. Fowler, H. Schmid and D. Kern, Phys. Rev. Lett. 61, 2801 (1988).

[20] M. Hilke, D. C. Tsui, M. Grayson, L. N. Pfeiffer and K. W. West, cond-mat/0104553 (2001).

[21] B. J. van Wees, E. M. M. Willems, L. P. Kouwenhoven, C. J. P. M. Harmans, J. G. Williamson, C. T. Foxon and J. J. Harris, Phys. Rev. B 39, 8066 (1989).

[22] S. Komiyama, H. Hirai, S. Sasa and S. Hiyamizu, Phys. Rev. B 40, 12566 (1989).

[23] G. Müller, D. Weiss, A. V. Khaetskii, K. v. Klitzing, S. Koch et al., Phys. Rev. B 45, 3932 (1992).

[24] The data shown in Fig. 3 were taken on a sample with a slightly different geometry, having two separate inner etched regions. In this case, the fully equilibrated resistances in units of $h / e^{2}$ are $1 / 4,1 / 2,3 / 4$, and 1 for the configurations $R_{73,85}, R_{83,75}, R_{75,83}$ and $R_{85,73}$, respectively.

[25] R. Wildfeuer, diploma thesis LMU Munich, unpublished (1999).

[26] A. A. Shashkin, A. J. Kent, P. A. Harrison, L. Eaves and M. Henini, Phys. Rev. B 49, 5379 (1994).

[27] S. M. Sze, Physics of Semiconductor Devices, J. Wiley \& Sons, 2nd Ed. (1981).

[28] S. Komiyama, H. Hirai, M. Ohsawa, Y. Matsuda, S. Sasa and T. Fujii, Phys. Rev. B 45, 11085 (1992).

[29] T. Machida, H. Hirai, S. Komiyama, T. Osada and Y. Shiraki, Phys. Rev. B 54, 14261 (1996).

[30] A. Würtz, diploma thesis LMU Munich, unpublished (2001).

[31] B. W. Alphenaar, P. L. McEuen, R. G. Wheeler and R. N. Sacks, Phys. Rev. Lett. 64, 677 (1990).

[32] H. Hirai, S. Komiyama, S. Fukatsu, T. Osada, Y. Shiraki and H. Toyoshima, Phys. Rev. B 52, 11159 (1995). 